\title{
The Impact of Dispersion Fluctuation on the Optimization of Parametric Wavelength Exchange
}

\author{
Mengzhe Shen, Xing Xu, Ngai Wong, T. I. Yuk, and Kenneth K. Y. Wong \\ ${ }^{1}$ Department of Electrical and Electronic Engineering, the University of Hong Kong, Hong Kong, China \\ Phone: +(852) 90646587, Fax: +(852) 25598738, Email: mzshen@eee.hku.hk
}

\begin{abstract}
The optimized parameters of wavelength exchange vary from fiber to fiber due to zero-dispersion wavelength fluctuation. This problem can be solved by reducing wavelength separation between signal and idler at the expense of reduced tunability.
\end{abstract}

Parametric wavelength exchange (PWE) relies on fourwave mixing (FWM) phenomenon, where a signal wavelength at $\lambda_{\text {signal }}$ and an idler wavelength at $\lambda_{\text {idler }}$ exchange their power periodically while two strong pumps at $\lambda_{p 1}$ and $\lambda_{p 2}$ are co-propagating in highlynonlinear dispersion-shifted fibers (HNL-DSF) [1]. Four waves are arranged symmetrically with respect to the zero-dispersion wavelength (ZDW) $\lambda_{0}$ of the fiber, such that the angular frequencies $\omega_{i},(i=1-4)$ satisfy $\omega_{1}+$ $\omega_{4}=\omega_{2}+\omega_{3}=2 \omega_{0},\left(\omega_{1}<\omega_{2}<\omega_{3}<\omega_{4}\right)$, where $\omega_{j}(j=1-4)$ are respectively the frequencies of the pump $\# 1, \# 2$, signal, and idler waves, and $\omega_{0}$ corresponds to the $\lambda_{0}$ of the fiber. However, the manufacturing process of a fiber inevitably introduces core diameter variation, which causes ZDW fluctuations along the fiber. Since FWM is sensitive to phase mismatch among the interacting waves, it is important to know how the selection of pump wavelengths affects the PWE performance under the condition of ZDW variations. We have presented recently an optimization approach to increase the conversion ratio (CR) of PWE with the consideration of ZDW fluctuation [2]. One major drawback of it is arisen from the fact that the CRs after the PWE process vary considerably from fiber to fiber even though each fiber may have the same ZDW on average. Since the outcome CRs of different fibers vary greatly, the optimization parameters attained for one fiber may not be able to apply to the others. In other words, it is likely that no guarantee can be made about the optimized operation of a PWE if optimized parameters rely on only one realization of ZDW distribution along the fiber.

In this paper, we will investigate the dependence of stochastic CR characteristics for a cluster of one hundred pieces of fibers with the same ZDW, such as mean and standard deviation, on the selection of pump and signal wavelengths. We can find that the fluctuation of CRs reduces when the two signals are close to each other. It gives rise to a hint that the previous mentioned optimization method is applicable in these regions, although a constraint on the wavelength separation should be imposed.

If we assume that the pump powers are not depleted,
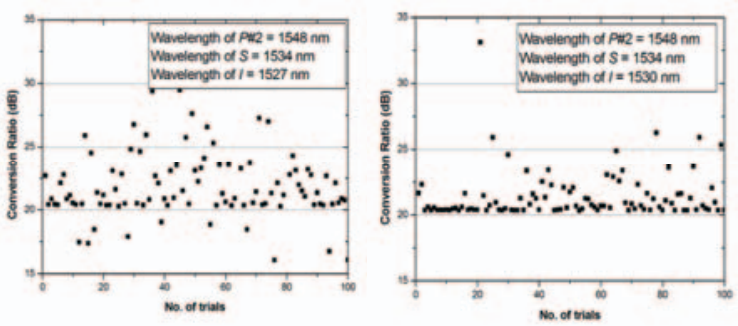

Fig. 1. Fiber-to-fiber variations in the PWE conversion ratio caused by random variations in ZDW along the fiber for the signal wavelength separation of $7 \mathrm{~nm}$ (left) and $4 \mathrm{~nm}$ (right).

the evolution of the signal $\left(B_{3}\right)$ and idler $\left(B_{4}\right)$ waves is governed by the following two equations [1]:

$$
\begin{aligned}
& \partial B_{3} / \partial z=i\left[2 \gamma \sqrt{P_{1} P_{2}} B_{4} / 3-\kappa B_{3} / 2\right] \\
& \partial B_{4} / \partial z=i\left[2 \gamma \sqrt{P_{1} P_{2}} B_{3} / 3+\kappa B_{4} / 2\right]
\end{aligned}
$$

Where $P_{1}$ and $P_{2}$ are pump powers respectively at frequencies $\omega_{1}$ and $\omega_{2}, \gamma$ is the nonlinear coefficient of the fiber, $\kappa=\Delta \beta+\gamma\left(P_{1}-P_{2}\right) / 3$ describes the total phase mismatch, and $\Delta \beta=\beta\left(\omega_{1}\right)+\beta\left(\omega_{4}\right)-\beta\left(\omega_{2}\right)-\beta\left(\omega_{3}\right)$ is the wave-vector phase mismatch related to the dispersion parameters as

$$
\Delta \beta=2 \sum_{m=1}^{\infty} \frac{\beta_{2 m}}{2 m !}\left[\left(\Delta \omega_{4}\right)^{2 m}-\left(\Delta \omega_{3}\right)^{2 m}\right]
$$

In which $\Delta \omega_{3}=\omega_{3}-\omega_{0}, \Delta \omega_{4}=\omega_{4}-\omega_{0}, \beta_{2 \mathrm{~m}}$ denotes the $2 m^{\text {th }}$ derivative of $\beta$ with respect to frequency around the ZDW as

$$
\beta_{2} \approx \beta_{3}\left[\left(\omega_{2}+\omega_{3}\right) / 2-\omega_{0}\right]+\beta_{4}\left[\left(\omega_{2}+\omega_{3}\right) / 2-\omega_{0}\right]^{2} / 2
$$

To gain better insight into $\Delta \beta$, we limit the summation of Eq. (3) to $m=1,2$.

When the ZDW is constant along the fiber, in theory, the exchanged wavelengths can be arbitrary by simply tuning the pump wavelengths correspondingly, such that $\kappa$ remains close to zero over a relatively broad spectral range. Random variations in the ZDW cause $\beta_{2}$ to vary randomly along the fiber, and it becomes difficult to maintain $\kappa=0$. As FWM is sensitive to local phase mismatch, the CRs reduce considerably even if the fiber and other design parameters (such as average ZDW, pump powers and wavelengths, strength of nonlinear coefficient, etc.) are otherwise optimized when ZDW varies randomly along the fiber even by a small amount of $1 \mathrm{~nm}$. Mathematically, random ZDW variations along the fiber render $\kappa$ random and transform (1) and (2) into two stochastic differential equations with multiplicative noise whose solution generally requires a numerical 


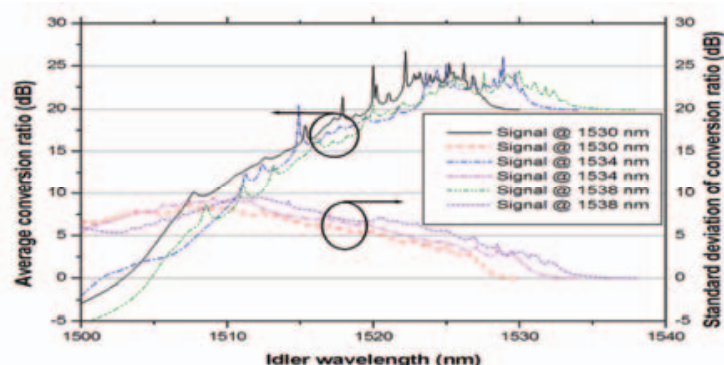

Fig. 2. The dependence of average $\mathrm{CR}$ and standard deviation for 100 realizations of fiber-to-fiber variation for different signal wavelength on idler wavelength.

approach $[3,4]$. Each realization of the random process corresponds to one fiber with a specific form of ZDW variations. PWE made by using different fiber pieces from the same spool will exhibit different CR because each corresponds to a different realization of the stochastic process. Such fiber-to-fiber variations in the CR can be predicted by solving (1) and (2) numerically. We consider a PWE made using 1000-m HNL-DSF ( $\gamma=$ $11 \mathrm{~W} / \mathrm{km}$ ) for which the average ZDW is $1541 \mathrm{~nm}$ with dispersion parameters $\beta_{3}=100 \mathrm{ps}^{3} / \mathrm{m}$ and $\beta_{4}=0.1 \mathrm{ps}^{4} / \mathrm{m}$. One of the signals is fixed at $1534 \mathrm{~nm}$ and the idler wavelength is a variable. The PWE is assumed to be pumped at $1548 \mathrm{~nm}$ (pump \#2) and a wavelength approximately symmetric with the idler wavelength with respect to the ZDW (pump \#1) with a power of $0.21 \mathrm{~W}$ at each wavelength. We assume that ZDW fluctuations follow a Gaussian distribution with a standard deviation of $1 \mathrm{~nm}$ and a correlation length of $5 \mathrm{~m}$. Fig. 1 (a) and (b) show the CRs for 100 realizations of the random process for signal and idler wavelength separation equals to 7 , and $4 \mathrm{~nm}$, respectively. It is evident that if the separation is small, the CRs will converge. A closer look at Eq. (3) reveals why it is necessary to reduce the wavelength separation to retain the CR uniformity. In this equation, ZDW appears only through $\beta_{2}$. When the signal wavelength is close to the idler, $\Delta \beta$ nearly vanishes regardless of the ZDW. However, as signal wavelength moves away, fluctuations in $\Delta \beta$ increase and become maximum for $\omega_{4}=\omega_{0}$. Reducing the signal and idler separation guarantees that fluctuations in $\Delta \beta$ and the CRs can be kept below a reasonable value.

In Fig. 2, we explore this issue further by fixing the wavelength of signal and pump \#2 and tune the idler wavelength from $1500 \mathrm{~nm}$ to signal wavelength. The wavelength of pump \#1 is tuned accordingly. The signal wavelengths are chosen at 1530,1534 , and $1538 \mathrm{~nm}$ for comparison. Two features are investigated in particular, one of which is the average CR, the other is the standard deviation of the CRs for 100 realizations of the random process. It can be observed that for signal wavelength at $1538 \mathrm{~nm}$, its standard deviation can maintain at nearly zero over longer idler wavelength span than that for signal wavelength at $1530 \mathrm{~nm}$. The explanation for this phenomenon rises from Eq. (4). We can also derive the conclusion from Fig. 2 that since the CR variation for different fiber-to-fiber realization is neglected when signal and idler wavelengths are close to each other, the optimization parameters achieved for one realization can be applied to every other realization. In other words, the concern mentioned in the introduction part of this paper can be resolved as long as the constraint about signal and idler wavelength separation remains.

Therefore, we use the same optimization technique based on the numerical convex programming solver Gloptipoly [5] in [2] to look for the wavelength of pump \#2 that maximize the average CR and minimize the their standard deviation. Based on to the discussion above, this objective equals to minimizing the mean square of $\Delta \beta$ along the fiber length for any single fiber-to-fiber variation, and the problem can be formulated as the following optimization problem,

$$
\text { Minimize } \frac{1}{L} \int_{0}^{L} \Delta \beta^{2}\left(\omega_{0}(z), \omega_{P 1}, \omega_{P 2}\right) d z
$$

Subject to

$$
\begin{aligned}
& \omega_{P 1 L} \leq \omega_{P 1} \leq \omega_{P 1 U} \\
& \omega_{P 2 L} \leq \omega_{P 2} \leq \omega_{P 2 U} \\
& \omega_{P 1}-\omega_{P 2}=\omega_{s}-\omega_{i}
\end{aligned}
$$

Table 1 shows the optimized wavelength arrangements and improved CR. The simulation parameters are the same as those in Fig. 2 except that the wavelengths of signal and idler are fixed close enough in order to avoid CR fluctuations.

\begin{tabular}{|l|l|l|}
\hline Input Waves & Unoptimized & Optimized \\
\hline Pump \#2 & $1548.12 \mathrm{~nm}$ & $1547.95 \mathrm{~nm}$ \\
\hline Signal & $1534 \mathrm{~nm}$ & $1534 \mathrm{~nm}$ \\
\hline Idler & $1529 \mathrm{~nm}$ & $1529 \mathrm{~nm}$ \\
\hline
\end{tabular}

Table 1 Wavelengths of Pump \#2 under unoptimized and optimized arrangements.

In conclusion, effects of ZDW fluctuations on PWE are investigated analytically and numerically. It is found that the CRs vary considerably from fiber to fiber even though each fiber may have the same ZDW on average. It is found that the variation is reduced when the wavelength separation between signal and idler is small. In this situation, it gives rise to the validity that optimization parameters obtained for one realization can be applied to any other realization as long as the wavelength separation from signal and idler is small, which greatly relaxes the optimization procedure.

\section{Acknowledgment}

The work described in this paper was partially supported by grants from the research Grants Council of the Hong Kong Special Administrative Region, China (Project No. HKU 7172/07E and HKU 7179/08E).

\section{References}

[1] K. Uesaka, et.al, IEEE J. Selected Topics in Quantum Electron., 8, pp.560-568, 2002

[2] B. P. P. Kuo, et.al, in Proc. Opt. Electron. and Commun. Conf. (OECC), Taiwan, 2006

[3] F. Yaman, et.al, Photon.Tech.Lett. 16, pp.1292-1294, 2004.

[4] N. Wong, et.al, Optics Communications, 272, pp. 514-520, 2007.

[5] J. B. Lasserre, SIAM J. Optimizaiton, 11, pp. 796-817, 2001. 